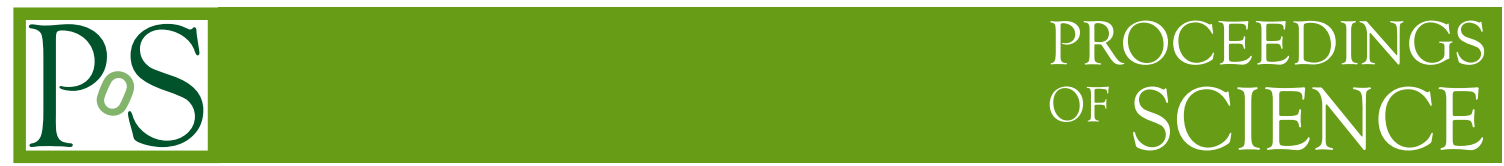

DESY-13-210, SFB/CPP-13-90, HU-EP-13/65

\title{
Looking at the gluon moment of the nucleon with dynamical twisted mass fermions
}

\author{
Constantia Alexandrou ${ }^{a b}$, Vincent $\operatorname{Drach}^{c}$, Kyriakos Hadjiyiannakou $^{a}$, Karl Jansen ${ }^{a c}$, \\ Bartosz Kostrzewa $^{d}$, Christian Wiese ${ }^{* c}$ \\ ${ }^{a}$ Department of Physics, University of Cyprus, P.O. Box 20537, 1678 Nicosia, Cyprus \\ ${ }^{b}$ Computation-based Science and Technology Research Center, Cyprus Institute, 20 Kavafi Str., \\ Nicosia 2121, Cyprus \\ ${ }^{c}$ NIC, DESY Zeuthen, Platanenallee 6, D-15738 Zeuthen, Germany \\ ${ }^{d}$ Humboldt-Universität zu Berlin, Institut für Physik, Newtonstraße 15, D-12489 Berlin, \\ Germany \\ E-mail: christian.wiese@desy.de
}

To understand the structure of hadrons it is important to know the PDF of their constituents, the quarks and gluons. In our work we aim to compute the first moment of the gluon PDF $\langle x\rangle_{g}$ for the nucleon. We follow two possible approaches in order to extract the gluon moment: the FeynmanHellmann theorem and a direct method with smearing of the gluon operator. We present preliminary results computed on $24^{3} \times 48$ lattices for the case where the Feynman-Hellman theorem is used and $32^{3} \times 64$ lattices for the direct method, employing $N_{f}=2+1+1$ maximally twisted mass fermions.

31st International Symposium on Lattice Field Theory LATTICE 2013

July 29 - August 3, 2013

Mainz, Germany

${ }^{*}$ Speaker. 


\section{Introduction}

From deep inelastic scattering (DIS) experiments it is known, see e.g. [1], that the nucleon is not a fundamental particle but consists of so-called partons as its constituents. The contribution of these partons to the nucleon momentum is described by a parton distribution function (PDF) $f_{p}(x)$, which is the probability to find a parton $p$ with a momentum fraction $x$. The first moment of the PDF $\langle x\rangle_{p}=\int x f_{p}(x) d x$ is the fraction of the total nucleon momentum carried by the parton. This implies then the energy-momentum sum rule $\sum_{p}\langle x\rangle_{p}=1$. The partons were eventually identified as the quarks and the gluons as the fundamental building block of hadrons. Thus, the energy-momentum sum rule of partons translates directly to a sum rule involving all quarks and the gluons,

$$
\sum_{q}\langle x\rangle_{q}+\langle x\rangle_{g}=1
$$

Further experimental input suggests that the contribution coming only from up- and down quarks does not add up to one [2]. Since it is expected that the heavier quarks will not significantly contribute to the average nucleon momentum [3], this implies that the gluons carry a large amount of the nucleon momentum, such that the sum rule of eq. (1.1) is satisfied.

Therefore, the computation of the gluon moment is necessary to fully understand the structure of the nucleon. However, at the moment, despite the fact that there are many results for the quark structure of the nucleon, see e.g. refs. [曰, 5], there are just a few results for $\langle x\rangle_{g}$ which are, moreover, only obtained from quenched computations [6, 7]. The work presented here aims at giving a first result from a computation on gauge configurations generated with light, strange and charm sea quarks.

We can access the gluon moment of a hadron via the matrix elements of the gluon operator:

$$
O_{\mu \nu}=-\operatorname{tr}_{c} G_{\mu \rho} G_{v \rho} .
$$

The matrix elements of this operator can be computed with a ratio of a three-point and a two-point function, where the sink time $t$ and the operator time $\tau$ are chosen properly.

$$
\frac{\langle h(p, t) \mathscr{O}(\tau) h(p, 0)\rangle}{\langle h(p, t) h(p, 0)\rangle} \stackrel{0 \ll \tau \ll t}{=}(\mathscr{O})_{h(p) h(p)}
$$

where $h(p, t)$ denotes a hadron with momentum $p$ at sink time $t$. The general matrix element of eq. (1.3) can be decomposed into several terms proportional to appropriate form factors, see e.g. the discussion in ref. [4]. The relevant form factor for our purpose is $A_{20}$, which can be related to the gluon moment. In order to proceed, we need to consider certain representations of the operator in eq. (1.2). Here we choose two of them

$$
\mathscr{A}_{i}=\mathscr{O}_{i 4}, \mathscr{B}=\mathscr{O}_{44}-\frac{1}{3} \mathscr{O}_{j j}
$$

The matrix elements of these operatoprs can be written in terms of the gluon moment as

$$
\left(\mathscr{A}_{i}\right)_{N(p) N(p)}=-i p_{i}\langle x\rangle_{g}, \quad(\mathscr{B})_{N(p) N(p)}=\left(m_{N}+\frac{2}{3 E_{N}} \vec{p}^{2}\right)\langle x\rangle_{g} .
$$

Both operators have certain drawbacks. The operator $\mathscr{A}_{i}$ can only be taken when a non-zero momentum is injected. It is known that the computation of momentum dependent operator matrix 
elements result in a larger noise-to-signal ratio than a momentum-zero computation, which is possible for operator $\mathscr{B}$.

In case of the operator $\mathscr{B}$ there is a subtraction of two terms which are similar in magnitude. This can be understood from the lattice version of the operator, expressed in terms of plaquettes:

$$
\mathscr{B}(t)=\frac{4}{9} \frac{\beta}{a} \sum_{x}\left(\sum_{i} \operatorname{tr}_{c}\left[U_{i 4}(x, t)\right]-\sum_{i<j} \operatorname{tr}_{c}\left[U_{i j}(x, t)\right]\right) .
$$

Here, one sees that the spatial and the temporal part of the plaquette, which are very similar in size, need to be subtracted, leading potentially again to a bad signal-to-noise behavior of the corresponding matrix element. The choice we made for the following discussion is nonetheless the operator $\mathscr{B}$ since it is directly accessible to us.

\section{Feynman-Hellman theorem}

One approach to extract the matrix elements of the gluon operator that was applied in [6] uses the Euclidean form of the Feynman-Hellman theorem. If one introduces some operator $\lambda \mathscr{O}$ into the action of the system, the operator's matrix elements can be derived from the derivative of the energy of the state with respect to $\lambda$.

$$
\frac{\partial E_{N}(\lambda)}{\partial \lambda}=\left(: \frac{\partial \hat{S}(\lambda)}{\partial \lambda}:\right)_{N(p) N(p), \lambda}
$$

Here $: \ldots$ : means that the vacuum expectation value of the operator has to be subtracted. For the purpose of calculating the three-point function for the gluon operator we modify the Wilson gauge action as

$$
S(\lambda)=\frac{1}{3} \beta(1+\lambda) \sum_{i} \operatorname{tr}_{c}\left[1-U_{i 4}\right]+\frac{1}{3} \beta(1-\lambda) \sum_{i<j} \operatorname{tr}_{c}\left[1-U_{i j}\right]
$$

Note that $\lambda=0$ corresponds to the standard Wilson plaquette gauge action. When applying eq. (1.5), (1.6) and (2.1) one can relate the derivative of the nucleon energy to $\langle x\rangle_{g}$.

$$
\left.\frac{\partial E_{N}}{\partial \lambda}\right|_{\lambda=0}=-\frac{3}{2}\left(m_{N}+\frac{2}{3 E_{n}} \vec{p}^{2}\right)\langle x\rangle_{g}
$$

There is no subtraction of the vacuum expectation value here, because utilizing lattice rotational symmetry it can be shown that the expectation value of the operator in eq. (1.6) is zero. When computing the nucleon mass at zero momentum, the relation can be simplified as:

$$
\langle x\rangle_{g}=\left.\frac{2}{3 m_{N}} \frac{\partial m_{N}}{\partial \lambda}\right|_{\lambda=0} .
$$

In order to compute the nucleon mass for different, non-zero $\lambda$ values, new gauge ensembles had to be generated. In addition, due to the change of the gauge action, the hopping parameter $\kappa$ had to be re-tuned to its critical value for each ensemble, in order to regain the $\mathscr{O}(a)$ improvement.

We have performed preliminary tests on small lattices with heavy quark masses to keep the computational effort affordable. The simulations were carried out with $24^{3} \times 48$ lattices and $N_{f}=$ 
$2+1+1$ flavors of maximally twisted mass fermions. We employed $\beta=1.95$ which corresponds to a lattice spacing of $a \approx 0.078 \mathrm{fm}$ and a twisted mass parameter $\mu=0.085$ which leads to a pion mass of $m_{P S} \approx 490 \mathrm{MeV}$. As gauge action we used the Iwasaki action, however the FeynmanHellman theorem was only applied to the Wilson part, i.e. the pure plaquette part, of the action.

Our results for three different $\lambda$ values on $\sim 200$ gauge configurations and the nucleon mass at $\lambda=0$ can be seen in Fig. 11.

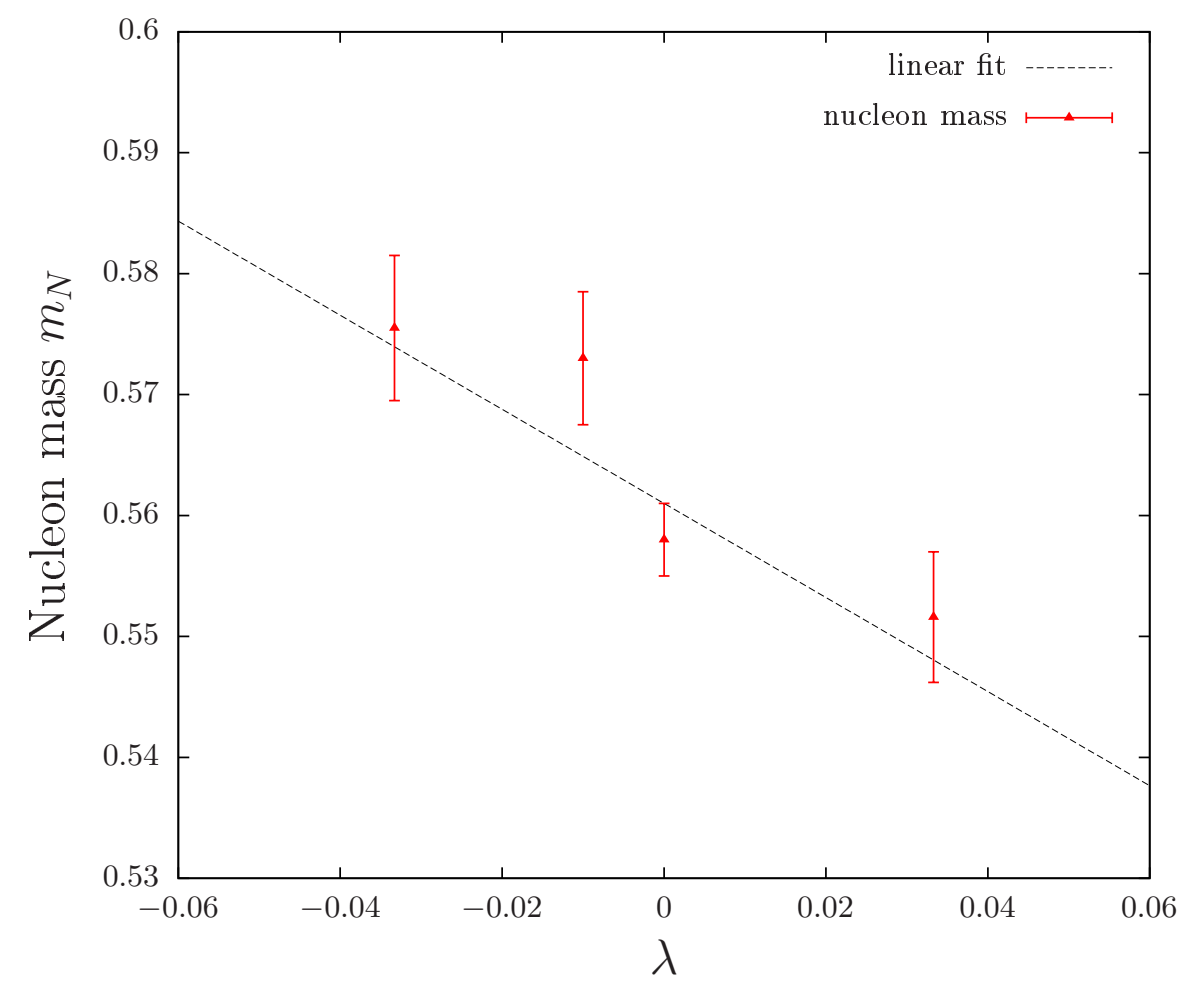

Figure 1: Dependence of the nucleon mass on the change of the gauge action (different $\lambda$ values). The slope of the fit can be related to the gluon moment.

We performed a linear fit in $\lambda$ to the data of the nucleon mass. The fact that the data shows a $\lambda$ dependence suggests that we can obtain a non-zero signal for the gluon moment. However, the error of the slope is rather large (about 30\%). The systematic error is probably even larger, because it is not known in which $\lambda$ region a linear fit is really justified. To study this systematic effect one would need to compute the nucleon mass with a smaller error for more $\lambda$ points than used here.

\section{Direct method}

An alternative, more straightforward method of computing the matrix element of eq. (1.3) is a direct approach, where, through performing the relevant Wick contractions, the three-point function can be expressed by a suitable combination of propagators and gauge links. For the gluon threepoint function this is actually a trivial task, because there are no quark fields in the gluon operator. Subsequently, there are no possible contractions between the gluon operator and the interpolating fields of the nucleon. The three-point function can, in fact, be written as a product of nucleon 
two-point functions and the gluon operator. For the zero momentum computation we get

$$
\frac{\left\langle[N(t) N(0)]_{p=0} \mathscr{B}(\tau)\right\rangle}{\left\langle N(t) N(0)_{p=0}\right\rangle} \stackrel{0 \ll \tau \ll t}{=} m_{N}\langle x\rangle_{g} .
$$

The advantage of this method is that we can reuse existing two-point functions and only have to compute the gluon operator on the very same configurations which requires little computational effort.

The following results were computed on a $32^{3} \times 64$ lattice with $N_{f}=2+1+1$ flavors of maximally twisted mass fermions. We set $\beta=1.95$, which corresponds to a lattice spacing of $a \approx 0.078 \mathrm{fm}$ and the twisted mass parameter $\mu=0.055$, which is a pion mass of $m_{P S} \approx 393 \mathrm{MeV}$. For the two-point function we used 16 different source positions on each gauge configuration which corresponds to 32 measurements, because we considered proton and neutron fields. The first results for a local gluon operator can be seen in the left panel of Fig. 国.
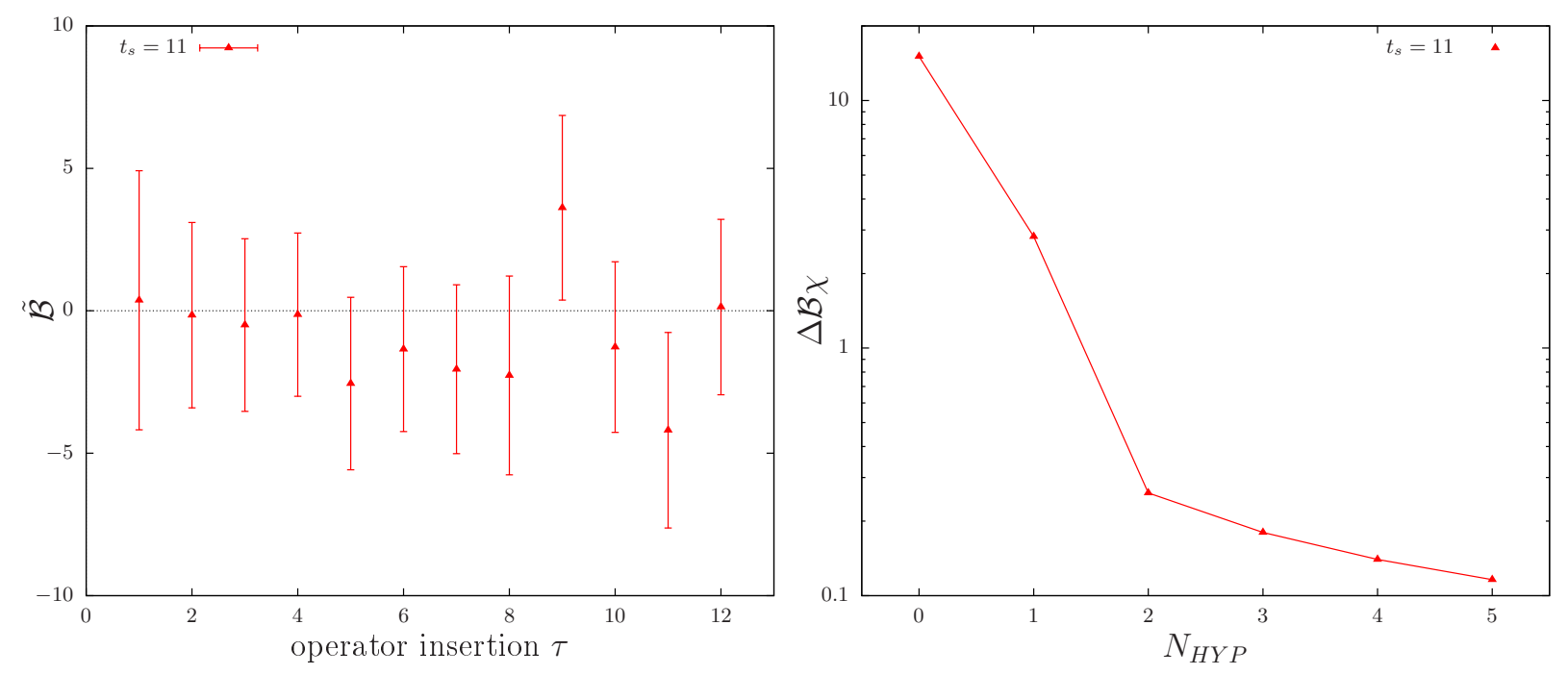

Figure 2: left: Nucleon matrix element for a local gluon operator for a source-sink separation of 11 and different operator insertion times $\tau$. right: Relative error of the nucleon matrix element for different HYPsmearing steps of the gluon operator.

Obviously, it is not possible to extract a signal, due to a large noise-to-signal ratio. A possible solution for this problem can be found in [8], where it is suggested to use HYP smearing [9] for the links in the gluon operator. We applied several steps of HYP smearing with parameters from [0] and present the relative error (noise-to-signal ratio) for the observable in the right panel of Fig. 2 .

We found a significant reduction of the noise-to-signal ratio with increasing number of HYP smearing steps. Thus, we subsequently applied five steps of HYP smearing.

On the left panel of Fig. B one can see the signal we got from a single source-sink separation, where $\mathscr{B}=\frac{4}{9} \beta \chi \tilde{\mathscr{B}}$ and $\chi$ is a normalization factor caused by using HYP smearing. We clearly got a non-zero value with a reasonable error of about $10 \%$. However, this signal could still be contaminated by excited state effects. This can be checked by computing the matrix element for different source-sink separations. On the right panel one can see that there are no strong excited states effects, because the plateau value seems to be stable for different sink time positions. 

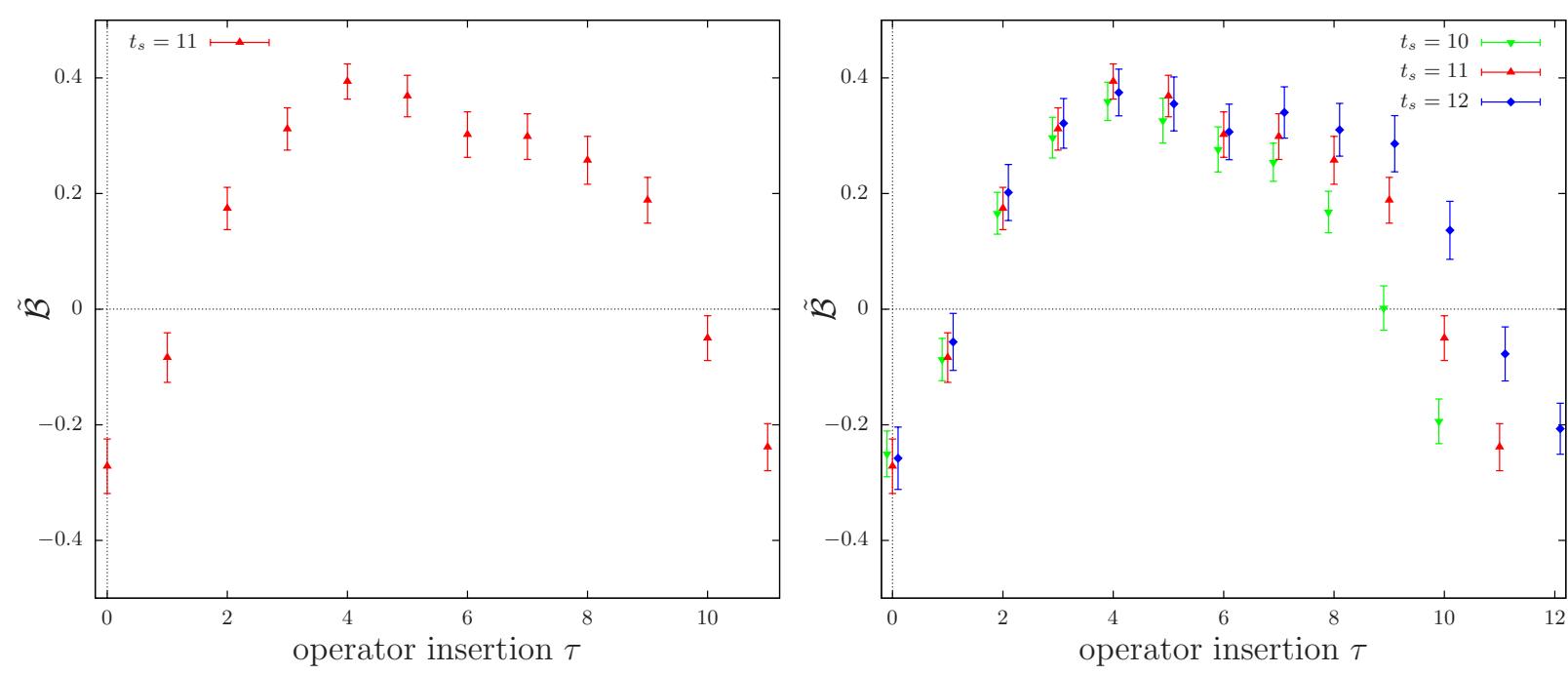

Figure 3: left: Nucleon matrix element for a HYP-smeared gluon operator for a source-sink separation of 11 and different operator insertion times $\tau$. right: The same matrix element for three different source-sink separations. $\mathscr{B}=\frac{4}{9} \beta \chi \tilde{\mathscr{B}}$

\section{Conclusion and outlook}

We presented two methods which potentially can be used to extract $\langle x\rangle_{g}$ on the lattice: The first method makes use of the Feynman-Hellman theorem and has the advantage of yielding a statistically significant signal for rather moderate statistics. However, the calculation needs dedicated simulations with different values of $\lambda$ to establish unambiguously the linear dependence of the results on $\lambda$. Furthermore, each simulation has to be tuned to a critical value of $\kappa$, in order to ensure automatic $\mathscr{O}(a)$ improvement. Therefore, overall, the computational cost associated with this method is large.

The second method directly computes the three-point function from which $\langle x\rangle_{g}$ can be extracted. In order to obtain a non-zero signal, one has to apply smearing on the gauge links entering the operator. Although one needs large statistics, one can use nucleon two-point functions computed for other observables and therefore the overall cost is small.

Our study therefore suggests that the direct method may be the method of choice to calculate this particular observable. Still, the Feynman-Hellman theorem could be used as a cross-check on ensembles where it is feasible to apply.

Another issue regarding the computation of the physical value of $\langle x\rangle_{g}$ is that the lattice matrix element needs to be renormalized. Since the gluon operator is a singlet operator it will mix with the quark momentum fraction $\langle x\rangle_{q}$. The relation between the renormalized and the bare values of both quantities is given by a $2 \times 2$ mixing matrix.

$$
\left(\begin{array}{c}
\langle x\rangle_{g}^{\overline{M S}} \\
\sum_{q}\langle x\rangle_{q}^{\overline{M S}}
\end{array}\right)=Z_{2 \times 2}\left(\begin{array}{c}
\langle x\rangle_{g}^{\text {bare }} \\
\sum_{q}\langle x\rangle_{q}^{\text {bare }}
\end{array}\right) .
$$

For $\langle x\rangle_{g}$ the relevant matrix elements are called $Z_{g g}$ and $Z_{q q}$ and the relation is

$$
\langle x\rangle_{g}^{\overline{M S}}=Z_{\text {bare gg }}^{\overline{M S}}\langle x\rangle_{g}^{\text {bare }}+\left[1-Z_{\text {bare qq }}^{\overline{M S}}\right] \sum_{q}\langle x\rangle_{q}^{\text {bare }} .
$$


As a first step we will compute these factors perturbatively. This will provide us with a first estimate of the factors and we will get insight in the renormalization process of this quantity. If we know the renormalization conditions, a non-perturbative renormalization can follow. Since the smearing of the operator should be included into the renormalization process, we will also try to use other smearing techniques for the lattice computation, i.e. HEX or stout smearing, which can be easier employed in a perturbative computation. Once the renormalization is complete we will be able to give the first physical result for $\langle x\rangle_{g}$ with fully active sea quarks.

The next step should be the computation of $\langle x\rangle_{g}$ at physical pion mass using the recently generated ensembles with $N_{f}=2$ twisted-mass-clover fermions [10]. For heavier quark masses the continuum limit for this quantity can be studied.

Furthermore, the result can be used for the determination of the gluon contribution to the nucleon spin, which at the moment is not known from the lattice. Moreover, it could also be possible to compute the gluon moment of other hadrons, e.g. the pion (cf. [8]).

\section{Acknowledgments}

We thank Carsten Urbach for discussion and help with the tmLQCD code [11], which has been used for the computations. Latest developlments for this code were also presented at this conference [12, 13]. This work has been supported in part by the DFG Sonderforschungsbereich/Transregio SFB/TR9-03. B.K. is supported by the National Research Fund, Luxembourg.

\section{References}

[1] R. Devenish and A. Cooper-Sarkar. Deep Inelastic Scattering. Oxford University Press, 2004.

[2] J. Blümlein, H. Böttcher and A. Guffanti, Nucl. Phys. B 774 (2007) 182 [hep-ph/0607200].

[3] A. D. Martin, W. J. Stirling, R. S. Thorne and G. Watt, Eur. Phys. J. C 63, 189 (2009) [arXiv:0901.0002 [hep-ph]].

[4] C. Alexandrou, M. Constantinou, S. Dinter, V. Drach, K. Jansen, C. Kallidonis and G. Koutsou, Phys. Rev. D 88 (2013) 014509 [arXiv:1303.5979 [hep-lat]].

[5] C. Alexandrou, M. Constantinou, S. Dinter, V. Drach, K. Hadjiyiannakou, K. Jansen, G. Koutsou and A. Strelchenko et al., PoS LATTICE 2012 (2012) 163 [arXiv:1211.4447 [hep-lat]].

[6] R. Horsley et al. [QCDSF and UKQCD Collaborations], Phys. Lett. B 714 (2012) 312 [arXiv:1205.6410 [hep-lat]].

[7] K. F. Liu, M. Deka, T. Doi, Y. B. Yang, B. Chakraborty, Y. Chen, S. J. Dong and T. Draper et al., PoS LATTICE 2011 (2011) 164 [arXiv:1203.6388 [hep-ph]].

[8] H. B. Meyer and J. W. Negele, Phys. Rev. D 77 (2008) 037501 [arXiv:0707.3225 [hep-lat]].

[9] A. Hasenfratz and F. Knechtli, Phys. Rev. D 64 (2001) 034504 [hep-lat/0103029].

[10] A. Abdel-Rehim et al. [ETM Collaboration], PoS LATTICE 2013 (2013) 264, to be published.

[11] K. Jansen and C. Urbach, Comput. Phys. Commun. 180 (2009) 2717 [arXiv:0905.3331 [hep-lat]].

[12] C. Urbach, PoS LATTICE 2013 (2013) 414, to be published.

[13] B. Kostrzewa, PoS LATTICE 2013 (2013) 416, to be published. 